\title{
Frustrated phase separation in two-dimensional charged systems
}

\author{
C. Ortix, ${ }^{1}$ J. Lorenzana, ${ }^{2,3,4}$ and C. Di Castro ${ }^{2,3}$ \\ ${ }^{1}$ Dipartimento di Fisica, Università di Lecce and INFN Sezione di Lecce, Via per Arnesano, 73100 Lecce, Italy. \\ ${ }^{2}$ Dipartimento di Fisica, Università di Roma "La Sapienza", P. Aldo Moro 2, 00185 Roma, Italy. \\ ${ }^{3}$ SMC-Istituto Nazionale di Fisica della Materia \\ ${ }^{4}$ ISC-Consiglio Nazionale delle Ricerche
}

(Dated: June 22, 2018)

\begin{abstract}
We study phase-separation frustrated (FPS) by the long-range Coulomb interaction in twodimensional electronic systems with emphasis to the case of a metallic and an insulating phase. In the mixed phase the system self-organizes in terms of mesoscopic inhomogeneities of one phase hosted by the other phase. We analyze the cases of circular drops and of alternating stripes. As a first approximation we consider the density inside each inhomogeneity as constant and in some cases we test the accuracy of this assumption by a more involved local density approximation. We find that the transition from the uniform phase to the frustrated phase-separated phase changes order depending upon its geometric arrangement. Contrary to what was found in three dimensional systems, there is no upper bound for the size of inhomogeneities. This difference stands on the different role of the long-range Coulomb interaction and screening in two and three dimensional systems. We conclude that two-dimensional systems are more prone to mesoscopic FPS.
\end{abstract}

PACS numbers: $64.75 .+\mathrm{g}, 71.10 . \mathrm{Hf}, 71.10 . \mathrm{Ca}$

\section{INTRODUCTION}

A variety of strongly correlated charged systems shows a strong tendency to phase separation (PS) 1.2.3.4.5.6.7.8.9.10.11.12.13.14 Often these system are two-dimensional (2D) like the two-dimensional electron gas (2DEG) $)^{9,10}$ or quasi two-dimensional like cuprates ${ }^{1,2}$ or some manganites $3,15,16,17,18,19$

Advances in local probe techniques have revealed the mesoscopic nature of the phase coexistence. For example cuprates phase separate into superconducting like regions and poorly metallic regions on the scale of $\sim 5 \mathrm{~nm} \sim$ 10 lattice constants, 11.12 .13 The regions have round like shapes indicating that surface energy has an important role.

Mesoscopic phase coexistence has also been reported in

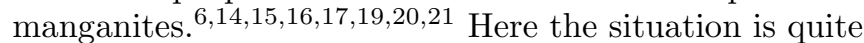
complex. Some systems have large insulating/metallic clusters with fractal like interfaces ${ }^{20}$ In this case phase separation is dominated by disorder effects stabilizing one or the other of the two phases very close in energy. On the other hand scanning tunneling spectroscopy in thin films have revealed filamentary and drop like metallic/insulating regions in the scale of tenths to thousand of nanometers with smooth surfaces indicating strong surface energies. In both cases percolation of the metallic regions is closely correlated to abrupt changes in transport 14.18 Finally neutron and X-ray scattering has revealed much smaller clusters, on the nanometer scale, in the bulk $6,16,17,19$

Using a local probe in the 2DEG, Ilani and collaborators 9,10 have shown that close to a puzzling metal-insulator transition $22,23,24$ the system becomes inhomogeneous at a mesoscopic scale. In addition the compressibility close to the transition, departs sharply from the predictions of an homogeneous electron $\operatorname{gas} \frac{9,10.25,26,27.28}{}$

Mesoscopic inhomogeneities are generally expected in systems with a density driven first order phase-transition in the presence of long-range forces. This phenomenon is well known in a variety of systems ${ }^{29}$ ranging from neutron stars 30 to spinodal decomposition hampered by elastic forces 31 and it is also related to the problem of domain formation in ferromagnetic systems. 32.33

In charged systems the phase coexistence phenomenon is hampered by the long-range Coulomb interaction (LRC). Indeed a macroscopic charge imbalance would imply an electrostatic energy cost which grows faster than the volume in the thermodynamic limit. Thus it has been proposed that the system should break in domains in order to guarantee large-scale neutrality $4,5,7,8,30,34,35,36,37$ In this way the charge is segregated over some characteristic distance but the average density at large distances is constant.

When the scale of the inhomogeneities is mesoscopic, one can perform a general analysis of FPS independently of the specific short-range interaction favoring $\mathrm{PS}$, in the same spirit of the Maxwell construction (MC) 4.5.30.34.35.36.37 The specific short-range interaction, however, will account for different physical situations with different coexisting phases. Due to this mesoscopic hypothesis the inhomogeneities can be treated as charged classical objects. Their size and their relative distance are determined by the competition between the LRC interaction and the interface boundary energy. These effects determine the total "mixing energy" i.e. the excess of energy with respect to the unfrustrated PS. To characterize the degree of frustration one can define a dimensionless parameter $\lambda$ given by the ratio of the characteristic mixing energy and the characteristic energy gain due to PS.

In $3 \mathrm{D}$ systems it was shown that the LRC interaction favors uniform phases. $34,35.36$ Indeed the coexistence re- 
gion shrinks as $\lambda$ increases and the uniform phases are stabilized at densities where the ordinary MC analysis would predict a PS state. In addition the size of the inhomogeneities in 3D systems has been shown to satisfy a "maximum size theorem" that says that inhomogeneities can not have all linear dimensions much larger than the screening length ${ }^{34.35 .36}$

In this work we consider mesoscopic frustrated phaseseparation for 2D electronic systems, that is electrons confined to a plane but subject to the 3D Coulomb interaction in the presence of a rigid background. Muratov, instead, has considered the case of a $d$-dimensional system immersed in the $d$-dimensional Coulomb interaction ${ }^{37}$ As a first approximation we neglect disorder effects and concentrate on the thermodynamic behavior in the clean limit. We analyze in detail the coexistence between a compressible phase (named "B") and an incompressible phase (named "A") corresponding to the physically relevant case of phase separation between a metal and an insulator. The results are easily generalizable to two compressible phases $\underline{38} \mathrm{~A}$ non-rigid background produces peculiar effects close to an energy level crossing of the electronic phases ${ }^{34.39 .40}$ and will be discussed elsewhere in the present context. ${ }^{41}$

We consider two different types of inhomogeneities: drops of one phase into the other phase and alternating stripes of each phase. We assume a uniform density inside each inhomogeneity. This uniform density approximation (UDA) is relaxed in Sec. [IV and tested in Appendix B against a more involved local density approximation (LDA). UDA is shown to be quite accurate for the evolution of global thermodynamic quantities.

As in $3 \mathrm{D}$ systems, the LRC interaction stabilizes the homogeneous metallic phase at densities where non frustrated systems would show PS .

In contrast to the 3D case, in $2 \mathrm{D}$ the density driven transition to the inhomogeneous state changes order depending upon the different geometric arrangement. Within our approximation, one has a second-order transition to a droplet state while the transition to the stripes geometry is always first-order like.

Moreover in 2D systems the size of the inhomogeneities is not limited by the screening length. As a result we find that 2D systems are more prone to mesoscopic FPS than the $3 \mathrm{D}$ systems.

\section{GENERAL ANALYSIS}

As in the 3D case we assume a separation of length scales. In many models on a lattice it is found that shortrange forces tend to phase separate the electrons between electron rich and electron poor regions 3.42 .43 .44 .45 .46 .47 We assume that the short range forces lead to a doublewell form for the energy of an hypothetical uniform phase as a function of density. We call $f_{A}$ and $f_{B}$ the energy densities close to each minimum, which define the bulk energy of the phases. Long-range forces prevent large scale phase separation leading instead to domain formation. As a simplified assumption we assume uniform densities $\left(n_{B}, n_{A}\right)$ for the inhomogeneities of each phase (UDA) with sharp interfaces (soft interfaces have been considered by Muratov ${ }^{37}$ ). This charge distribution is compensated by a rigid background of density $n$. The UDA will be relaxed in section IV

The free energy per unit "volume" of the mixed-phase reads:

$$
f=(1-\nu) f_{A}\left(n_{A}\right)+\nu f_{B}\left(n_{B}\right)+e_{e l}+e_{\sigma}
$$

where $\nu$ denotes the volume fraction of the B-phase $\left(V_{B} / V\right), e_{e l}$ represents the LRC interaction energy density and $e_{\sigma}$ is the "surface" energy density. (Here and below, in order to keep a common nomenclature with the 3D case, we call "volume" a quantity with units of length squared and "surface" a quantity with units of length). Due to charge neutrality the global charge density of electrons has to compensate the charge density of the background $e n$. This leads to the following neutrality constraint:

$$
n=(1-\nu) n_{A}+\nu n_{B}
$$

To proceed further we assume specific geometries for the inhomogeneities. We will consider the competition among the two following geometries: $i$ ) drops (disks) of one phase surrounded by the other phase and $i$ i) a periodic structure of alternating stripes of the two coexisting phases.

For the drops we divide the system in cells of radius $R_{c}$ enclosing one domain of the B-phase with radius $R_{d}$. Similarly for the stripes the cell has width $2 R_{c}$ and contains a B-phase stripe of width $2 R_{d}$. The volume fraction is related to these characteristic lengths by:

$$
\nu=\left(\frac{R_{d}}{R_{c}}\right)^{\alpha} \quad \alpha=1,2
$$

where for $2 \mathrm{D}$ systems $\alpha=1,2$ for the stripe geometry and the drop geometry respectively. In the latter case the cells are slightly overlapping with: $\pi R_{c}^{2} N_{d}=V\left(N_{d}\right.$ indicates the number of cells in the system while $V$ is the total volume). For the stripe geometry $V=2 R_{c} L N_{d}$ where $L$ indicates the length of the stripes. The total surface energy per unit volume can be parameterized by a quantity $\sigma$ with dimensions of energy per unit surface (actually length in 2D). It reads:

$$
e_{\sigma}=\sigma \frac{N_{d} \Sigma_{d}}{V}
$$

where $\Sigma_{d}$ is the surface of the domain interface inside one cell with $\Sigma_{d}=2 \pi R_{d}$ and $\Sigma_{d}=2 L$ for the drops and the stripes respectively. For inhomogeneities of the B-phase hosted by the A-phase, $e_{\sigma}$ can be written as:

$$
e_{\sigma}=\frac{\sigma}{R_{c}} \alpha \nu^{\frac{\alpha-1}{\alpha}}
$$


The operation: $A \leftrightarrow B, \nu \leftrightarrow 1-\nu$ was named "phase exchange" in Ref. 34. Within the UDA the energy should not change under this operation ${ }^{34}$ For the stripe geometry case the surface energy does not depend on $\nu$ and Eq. (2) already preserves the phase-exchange symmetry. On the contrary for the drop geometry Eq. (2) is appropriate only for small $\nu$. In fact for intermediate volume fractions the drops should deform and for $\nu \simeq 1$ the Bphase should represent the host in which drops of the Aphase are immersed. Eq. (2), instead, violates this phase exchange symmetry because it involves drops of B-phase in both cases. We can define a "symmetrized" interpolating form that is correct at the two extremes $(\nu \simeq 0$ and $\nu \simeq 1$ ):

$$
e_{\sigma}=\frac{\sigma}{R_{c}} \alpha[\nu(1-\nu)]^{\frac{\alpha-1}{\alpha}}
$$

This is enough for our proposal because we can anticipate that drops are stable only in a narrow region close to $\nu \simeq 0$ and $\nu \simeq 1$ (c.f. Fig. 1).

The long-range Coulomb interaction energy is computed in the Appendix $\mathrm{A}$ by dividing the systems in neutral Wigner-Seitz cells. For stripes the electrostatic energy is computed numerically.

The electrostatic energy contribution due to the interaction between different cells can be neglected for volume fractions close to $\nu \sim 0$ and $\nu \sim 1$. In these limits, in fact, the inhomogeneities are far from each other and the interaction becomes irrelevant. We show below that this is indeed a reasonable approximation in the full range of volume fractions. As discussed in Appendix $\mathrm{A}$ we expect this approximation to be even more accurate for the drop geometry. Therefore for further analytical computations we use the approximate expression derived in Appendix $\mathrm{A}$ for both geometries:

$$
\begin{gathered}
e_{e l}=\frac{e^{2}}{\varepsilon_{0}}\left(n_{B}-n_{A}\right)^{2} R_{c} \frac{8}{3}[\nu(1-\nu)]^{\frac{3}{2}} \quad \text { Drops } \\
e_{e l}=\frac{e^{2}}{\varepsilon_{0}}\left(n_{B}-n_{A}\right)^{2} R_{c} 2[\nu(1-\nu)]^{2}[-\log \nu(1-\nu)]
\end{gathered}
$$

Stripes (5)

Here $\varepsilon_{0}$ is the static dielectric constant. For the drop geometry the electrostatic energy has been symmetrized similarly to the surface energy.

The bulk free energies $f_{A}$ and $f_{B}$ appearing in Eq. (11) do not depend upon $R_{c}$. The cell radius is therefore determined by minimizing the mixing energy $e_{m}=e_{e l}+$ $e_{\sigma}$ at fixed $\nu$. For the two geometries we have:

$$
R_{c}=\left(\frac{\sigma \varepsilon_{0}}{e^{2}\left(n_{B}-n_{A}\right)^{2}}\right)^{\frac{1}{2}} \frac{4 \sqrt{\nu(1-\nu)}}{u(\nu)}
$$

for the drops and

$$
R_{c}=\left(\frac{\sigma \varepsilon_{0}}{e^{2}\left(n_{B}-n_{A}\right)^{2}}\right)^{\frac{1}{2}} \frac{2}{u(\nu)}
$$
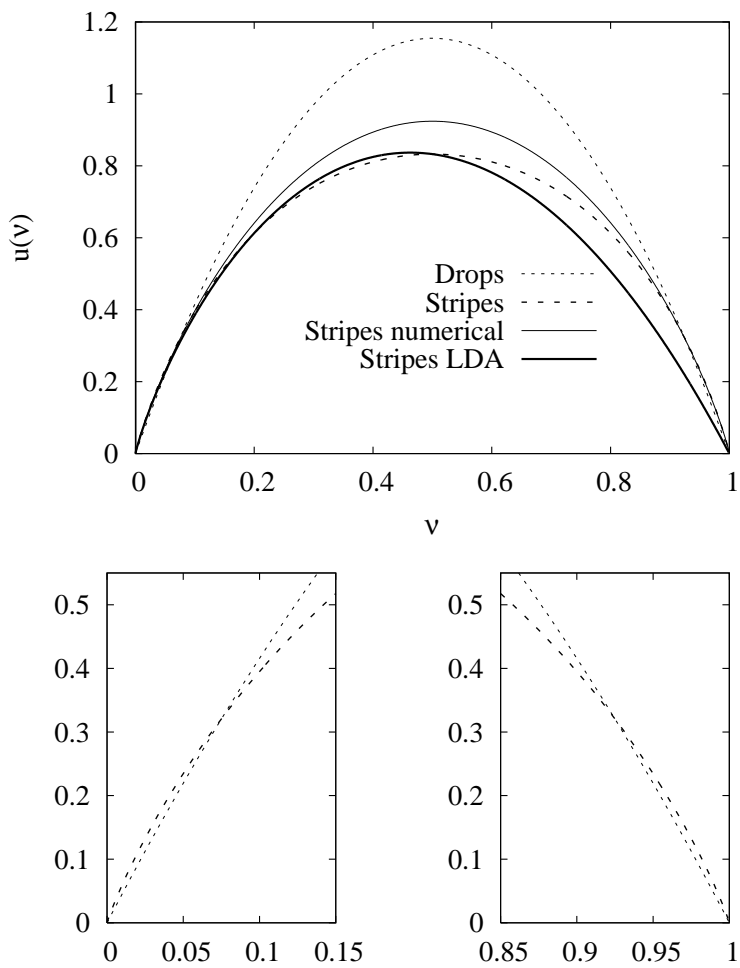

FIG. 1: Top: Approximate $u$ functions parameterizing the mixing energy for the drops [Eq. (9)] and the stripes [Eq. (10)]. For the latter geometry we also provide the numerically evaluated expressions taking into account the electrostatic interaction energy (see Appendix $[$ ), and the LDA (see Appendix. B] in order to test our approximations. Bottom: Expanded scale for $\nu \simeq 0$ and $\nu \simeq 1$ : the drop geometry introduces a lower mixing energy contribution.

for the stripes.

Notice that the exponent could be anticipated from dimensional analysis. For arbitrary dimensionality $d$ of the system we have:

$$
R_{c} \sim\left(\frac{\sigma \varepsilon_{0}}{e^{2}\left(n_{B}-n_{A}\right)^{2}}\right)^{\frac{1}{d}}
$$

times a function of $\nu$.

Once $R_{c}$ has been eliminated the mixing energy can be put in a common expression together with the $3 \mathrm{D}$ case of Ref. 34 3536 as:

$$
e_{m}=\left(\frac{\sigma^{d-1} e^{2}\left(n_{B}-n_{A}\right)^{2}}{\varepsilon_{0}}\right)^{\frac{1}{d}} u(\nu)
$$

where:

$$
u(\nu)=\frac{8}{\sqrt{3}} \nu(1-\nu)
$$

for 2D drops, while:

$$
u(\nu)=2 \sqrt{2} \nu(1-\nu)[-\log \nu(1-\nu)]^{\frac{1}{2}}
$$


for $2 \mathrm{D}$ stripes. The corresponding functions in the $3 \mathrm{D}$ case can be found in Ref. 34. The function $u(\nu)$ encodes all the information about the geometry and it is represented in Fig. 1 for the 2D case.

We see from Fig. 1 that only close to $\nu \sim 0$ and $\nu \sim 1$ the two geometries compete, since for intermediate volume fractions the stripe geometry is stabilized. The interplay among the two states is therefore well described by the analytical approximation for the electrostatic energy Eqs. (4),(5). Comparing the two curves for the stripe geometry we see that the use of the exact numerical expression will only produce small changes in physical quantities for $\nu \sim 1 / 2$ but cannot change the qualitative behavior. Thus, our use of the analytical expressions (Eqs. (4), (15)) of $e_{e l}$ is justified.

We minimize the total free energy per unit volume with respect to $n_{B}-n_{A}$ and $\nu$ as in Ref. 34 and obtain the coexistence equations for both $2 \mathrm{D}$ and $3 \mathrm{D}$ systems:

$$
\begin{aligned}
\mu_{B}-\mu_{A}= & -\frac{2}{d}\left(\frac{\sigma^{d-1} e^{2}}{\varepsilon_{0}}\right)^{\frac{1}{d}} \frac{n_{B}-n_{A}}{\left|n_{B}-n_{A}\right|^{2-\frac{2}{d}}} \frac{u(\nu)}{\nu(1-\nu)} \\
p_{B}-p_{A}= & {\left[n+\left(n_{B}-n_{A}\right)(1-2 \nu)\right]\left(\mu_{B}-\mu_{A}\right)+} \\
& \left(\frac{\sigma^{d-1} e^{2}\left(n_{B}-n_{A}\right)^{2}}{\varepsilon_{0}}\right)^{\frac{1}{d}} \frac{\partial u(\nu)}{\partial \nu}
\end{aligned}
$$

where

$$
\mu_{A, B}=\frac{\partial f_{A, B}}{\partial n_{A, B}}
$$

and the pressure is defined as:

$$
p_{A, B}=-f_{A, B}+\mu_{A, B} n_{A, B}
$$

We have neglected a possible dependence of $\sigma$ on density which contributes with additional terms (see Ref. 34).

Eq. (11) generalizes the ordinary Maxwell construction to the charged system case. The former $\left(\mu_{A} \equiv \mu_{B}\right.$ and $p_{A} \equiv p_{B}$ ) is recovered when $e \rightarrow 0$. The first of Eq. (11) states that the most dense phase has the lowest chemical potential. The difference of chemical potentials becomes particularly simple for $2 \mathrm{D}$ drops. In this case it is (within our approximations) constant and equal to $\pm 8 \sigma^{1 / 2} e /\left(3 \varepsilon_{0}\right)^{1 / 2}$.

\section{SEPARATION BETWEEN A COMPRESSIBLE AND AN INCOMPRESSIBLE PHASE}

Now we want to investigate an inhomogeneous state for a 2D system which involves an incompressible Aphase hereafter "the insulator" and a compressible Bphase hereafter "the metal" 38

This will be appropriate for example for a doped Mott insulator. The case of the $2 \mathrm{DEG}$ requires consideration of phases with negative compressibility and its full discussion will be presented elsewhere $\underline{\underline{41}}$

Since the insulator is incompressible and thus electrostatically inactive, we have to consider only the excess density of mobile electrons counting from the insulating state. Without loss of generality we set $n_{A} \equiv 0$ and $f_{A} \equiv 0$ for the insulator. In this way our density $n$ has the meaning of density deviation from the pure insulating phase. Also without loss of generality we consider $n>0$ so that the insulating phase is at low density and the metallic phase is at high density but our results apply equally well to the opposite case simply by changing $n \rightarrow-n$. With these conventions we are reduced to study the problem of the phase-separation between a metal at finite $n_{B}$ and "void" (playing the role of the insulator). Both phases are in the presence of a uniform background. In the present case the neutrality constraint reduce to: $\nu n_{B}=n$, while minimizing the free energy in the phase separated state the two Eqs. (11) reduce to a single equation for the B-phase pressure:

$$
p_{B}\left(n_{B}\right)=p_{m}
$$

where the function $p_{B}\left(n_{B}\right)$ is a property of the bulk phase [c.f. Eq. (12)] and

$$
p_{m}=\left(\frac{\sigma e^{2}}{\varepsilon_{0}}\right)^{\frac{1}{2}} n_{B}\left[\frac{\partial u(\nu)}{\partial \nu}-\frac{u(\nu)}{\nu}\right]
$$

Eq. (13) with the neutrality constraint determines the behavior of the local density and volume fraction as a function of $n$.

In the limit of $e \rightarrow 0$ we obtain the equation $p_{B}\left(n_{B}\right)=$ 0 which corresponds to Maxwell construction for the case of phase separation between a self-bound neutral fluid and vacuum.

When $e \neq 0, p_{m}$ represents the pressure due to the presence of the long-range Coulomb interaction and the surface energy, that we label "mixing pressure" and must be balanced by the B-phase pressure.

For the drop geometry one has:

$$
p_{m}=-\frac{8}{\sqrt{3}}\left(\frac{\sigma e^{2}}{\varepsilon_{0}}\right)^{\frac{1}{2}} n
$$

which depends on the global density of the system only.

Instead, in the stripes geometry the mixing pressure depends explicitly on the local density of the inhomogeneities:

$$
\begin{aligned}
p_{m}= & \frac{\left(\frac{\sigma e^{2}}{\varepsilon_{0}}\right)^{\frac{1}{2}}}{\left[-\ln \frac{n}{n_{B}}\left(1-\frac{n}{n_{B}}\right)\right]^{\frac{1}{2}}}[2 \sqrt{2} n+ \\
& \left.2 \sqrt{2} n \ln \left[\frac{n}{n_{B}}\left(1-\frac{n}{n_{B}}\right)\right]-\sqrt{2} n_{B}\right]
\end{aligned}
$$


The mixing pressure is negative for both geometries at all volume fractions. This means that the metallic phase is under "tensile stress" due to the long-range interaction. Thus the equilibrium density of the metal is lower than the density predicted by $\mathrm{MC}$ in the neutral case. For a discussion on the stability of a fractionated electronic fluid subject to a negative pressure see the Appendix B of Ref. 34 .

From the definition of the pressure one finds for the B-phase chemical potential:

$$
\mu_{B}=\frac{f_{B}\left(n_{B}\right)}{n_{B}}+\frac{p_{m}}{n_{B}}
$$

The last term is the contribution due to the frustrating forces. For the global chemical potential, $\mu=\partial f / \partial n$, we find:

$$
\mu=\frac{f_{B}\left(n_{B}\right)}{n_{B}}+\mu^{m i x}
$$

with the mixing contribution given by:

$$
\mu^{m i x}=\frac{\partial n_{B}}{\partial n} \frac{n}{n_{B}^{2}} p_{m}+\frac{d}{d n} e_{m}\left[n_{B}(n), \nu(n)\right] .
$$

We have provided the general equations for a FPS between an incompressible phase at $n_{A}=0$ and a compressible phase. To proceed further we need an explicit expression for the bulk free energy.

\section{A. Parabolic approximation for 2D metal free energy}

Now we solve our problem expanding the free energy of the B-phase in a parabolic approximation around the density at which, in absence of the long-range Coulomb interaction, the system would experience the transition to the phase-separated state. In other words we will label $n_{B}^{0}$ the density which satisfies the equation $p_{B}\left(n_{B}\right)=0$, $f_{B}^{0}$ the corresponding free energy $f_{B}^{0} \equiv f_{B}\left(n_{B}^{0}\right)$ and $\mu_{0}$ the corresponding chemical potential. These quantities are related by $f_{B}^{0}=\mu_{0} n_{B}^{0}$.

Using a Taylor expansion we can then write the Bphase free energy as follows:

$$
f_{B}\left(n_{B}\right)=\mu_{0} n_{B}+\frac{1}{2 \kappa_{B}}\left(n_{B}-n_{B}^{0}\right)^{2}
$$

where $\kappa_{B}=\left(\partial^{2} f / \partial^{2} n\right)^{-1}$ is proportional to the compressibility of the metallic phase.

The pressure of the B-phase is now:

$$
p_{B}\left(n_{B}\right)=\frac{1}{2 \kappa_{B}}\left(n_{B}^{2}-n_{B}^{0^{2}}\right)
$$

We introduce a dimensionless parameter $\lambda$ that measures the strength of the frustration due to the mixing energy effect. $\lambda$ can be defined as the ratio of the characteristic mixing energy (obtained from Eq. (8) without the
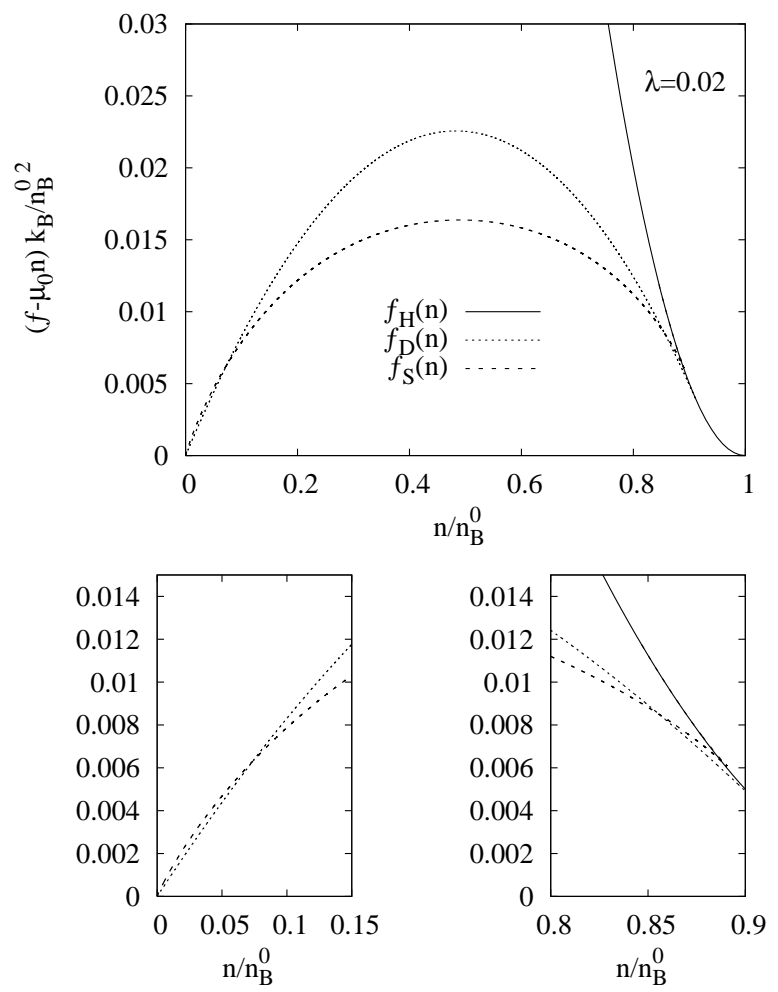

FIG. 2: Top: The free energy of the uniform metallic phase $f_{H}(n)$ compared with the FPS-state in the case of drops $\left[f_{D}(n)\right]$ and stripes $\left[f_{S}(n)\right]$ for $\lambda=0.02$. Bottom: Expanded scale in the density range where the geometries compete.

geometric factor $u(\nu))$ to the characteristic phase separation energy gain $\sim n_{B}^{02} / \kappa_{B}$ :

$$
\lambda=\frac{\kappa_{B}}{\left(n_{B}^{0}\right)^{2}}\left[\frac{\sigma^{d-1} e^{2}\left(n_{B}^{0}\right)^{2}}{\varepsilon_{0}}\right]^{\frac{1}{d}}
$$

$\lambda$ coincides apart from a numerical factor with the parameter introduced in Ref. 34 for $d=3$. In the following we measure the pressure and free energy densities in units of the characteristic phase-separation energy gain $\left(n_{0}^{B}\right)^{2} / k_{B}$ and all the densities in units of the characteristic MC density $n_{B}^{0}$. Thus we define $n_{B}^{\prime}=n_{B} / n_{B}^{0}$, $n^{\prime}=n / n_{B}^{0}, p_{B}^{\prime}=p_{B} \kappa_{B} / n_{B}^{0^{2}}$, the uniform $B$ phase energy density $f_{H}=f_{B} \kappa_{B} / n_{0}^{B} 2$ and the phase separated energy density $f_{D, S}=f \kappa_{B} / n_{0}^{B}{ }^{2}$. In our parabolic approximation the free energy densities read:

$$
\begin{aligned}
f_{H}\left(n^{\prime}\right)= & \mu_{0} n^{\prime} \frac{\kappa_{B}}{n_{B}^{0}}+\frac{1}{2}\left(n^{\prime}-1\right)^{2} \\
f_{D, S}\left(n^{\prime}, n_{B}^{\prime}\right)= & \mu_{0} n^{\prime} \frac{\kappa_{B}}{n_{B}^{0}}+\frac{n^{\prime}}{n_{B}^{\prime}} \frac{1}{2}\left(n_{B}^{\prime}-1\right)^{2}+ \\
& \lambda n_{B}^{\prime} u\left(\frac{n^{\prime}}{n_{B}^{\prime}}\right)
\end{aligned}
$$

The local density $n_{B}^{\prime}$ is determined by solving Eq. (13) with the left and right hand side given by Eq. (17) and Eqs. (14) and (15) respectively. 

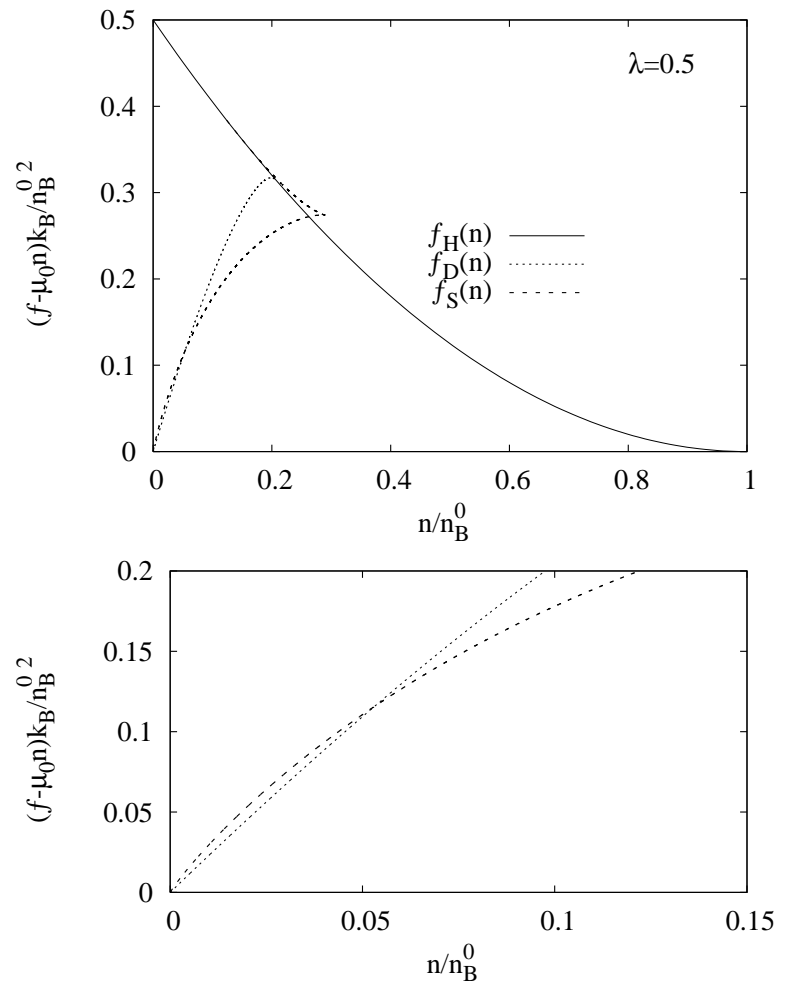

FIG. 3: Same as Fig. [2] for $\lambda=0.5$.

To decide the most stable geometry for the FPS and its stability against the uniform phase we have to compare the expressions in Eqs. (18) for different strengths of the LRC interaction. The first term in the free energies represents the $\mathrm{MC}$ free energy which is equal for all states and can be eliminated.

In Figs. 23 we plot the free energies with this term subtracted. We show the free energies of the uniform and the FPS-state in the presence of drops $\left(f_{D}\right)$ and stripes $\left(f_{S}\right)$ for two different values of the parameter $\lambda$. For small $\lambda$ (Fig. 2) the striped state introduces a lower energetic cost with respect to the drop geometry at intermediate global densities, while at low and high densities the two geometries compete. Increasing the global density one finds a first "geometrical" transition from a droplet state to a striped one at low density (left bottom panel) and a second "geometrical" transition from stripes to drops at high density (right bottom panel). For large $\lambda$ the situation is different (Fig. 3).The striped state results stable for both intermediate and high density and the two geometries compete only at low density (bottom panel) where there is a "geometrical" transition from the drops to the stripes as in the previous case.

Note that the free energies in the FPS-state for both geometries have a negative curvature and thus exhibit a negative electronic compressibility. This, however, does not imply necessarily an instability since the background contribution to the inverse compressibility, which is very large and positive ${ }^{34}$, should also be taken into account.
The range of global density where the FPS-state is stable depends upon $\lambda$. Furthermore inside the FPS-state one finds that the stable geometric arrangement changes both with the global density and the strength of the LRC interaction. This leads to construct a phase-diagram in the $n-\lambda$ plane (see Fig. 4). Given our initial choice we have two uniform phases, the metallic phase at high global densities and the insulating phase at $n=0$. For all $\lambda$ the two uniform phases are separated by FPS-states. The global density range of stability of the FPS, which for the unfrustrated case in absence of the LRC interaction is determined by $0<n^{\prime}<1$ (i.e. $0<n<n_{B}^{0}$ ), shrinks increasing $\lambda$. This is clearly due to the tendency of the long-range Coulomb interaction to stabilize the uniform state as in $3 \mathrm{D}$ systems 34 . Close to the insulator one finds metal drops and close to the uniform metal one finds circular voids in the metal. We will call the latter "the bubble state". Finally at intermediate densities one finds stripes. Increasing $\lambda$, the bubble state stability range shrinks and disappears above a value $\lambda^{*} \sim 0.1$ so that entering from the metallic uniform phase the FPS-state is made of stripe inhomogeneities. On the contrary the low density metallic drop state persists at all $\lambda$.

\section{Properties of the frustrated phase separated state}

We now discuss explicitly the physical properties of the FPS-state and the order of the various transitions. For the stripe state the relation for the local density as a function of global density is obtained in terms of $\nu$ in a parametric form. Then the volume fraction is obtained from the neutrality constraint. When the inhomogeneous phase has a drop geometrical arrangement (metal drop state and bubble state) the local density in the metal takes a particularly simple form. Solving Eqs. (13), (14), (17) with respect to $n_{B}$ we derive the Bphase local density in term of the global density:

$$
n_{B}^{\prime}=\sqrt{1-\frac{16}{\sqrt{3}} \lambda n^{\prime}}
$$

Obviously in the case $\lambda=0$ one recovers the MC solution $n_{B}=n_{B}^{0}$. Contrary to the MC the local density of the metallic inhomogeneities depends explicitly on and is a decreasing function of the global density $n$ of the system. Increasing the strength of the long-range interaction $\lambda$, this effect becomes stronger.

This behavior of the local density versus global density can be detected from local probes (like NQR and NMR). Physical quantities, which depend on the local density, will show an unexpected behavior: they will respond to an increase in density as if the density were decreasing. This effect has been discussed in connection with the Curie temperature in manganites 35.48

For the metal drop state at any $\lambda, n_{B}$ approaches the MC value $n_{B}^{0}$ going towards the homogeneous insulating phase, i.e. $n \rightarrow 0$ (Fig. 5). Furthermore the transition 

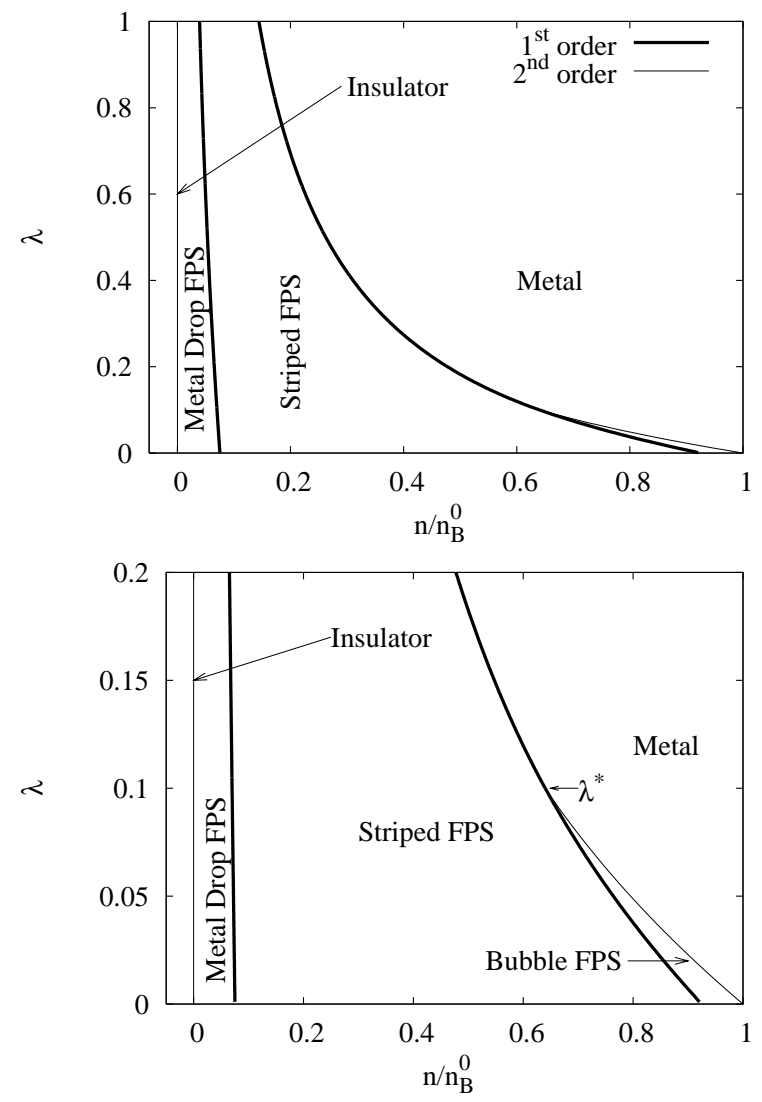

FIG. 4: Top: The phase-diagram in the $n-\lambda$ plane. Between the two uniform phases one finds the FPS-states. The transition from the insulator to the metal drop state is second order at all $\lambda$ (see Sec. IIIA 1), while the transition between the metal and the striped FPS-state at $\lambda>\lambda^{\star}$ is first order as well as the "geometrical" transitions. Bottom: Expanded scale for $\lambda \simeq \lambda^{\star}$. One finds a second-order transition from the metal to the "bubble" state.

from the FPS to the insulating-phase is second order like, since the volume fraction goes continuously to zero with sloop $1\left(\nu \sim n^{\prime}\right)$ (Fig. 6). From the same figure we see that for $\lambda=0.05<\lambda^{\star}$, the system goes from the uniform metal to the bubble state with the volume fraction for the insulating phase (given by $1-\nu$ ) which goes continuously to zero at the transition. Also the local density of the metallic regions is continuous (upper curve in Fig. 国) indicating that the transition is second-order like.

For $\lambda>\lambda^{\star}$ the region of stability of the bubbles close to $\nu \simeq 1$ disappears and the striped FPS-state appears at the transition with insulating inhomogeneities that have a finite volume fraction (Fig. 6). From these properties one can conclude that the transition is first-order in this case. The first order character of the transition can be understood from the behavior of the mixing pressure for the stripe case. From Eq. (15) one notes that in this case the mixing pressure has a divergence at $n_{B}=n$ which cannot be reached. Therefore one can not go continuously from the uniform metal to the stripe state.

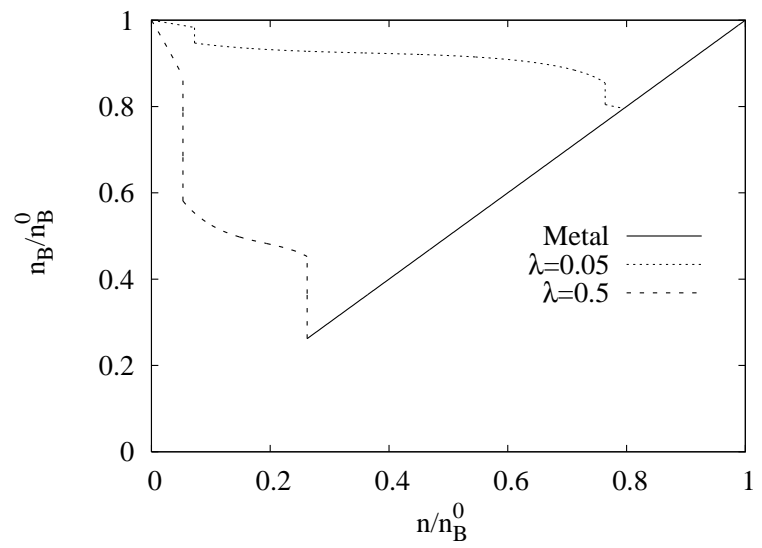

FIG. 5: The local density behavior vs. the global density for $\lambda=0.05,0.5$. In correspondence to the "geometrical" transitions and at the transition from the metal to the striped state $n_{B}$ shows a discontinuity reflecting their first-order nature. The segments close to the origin corresponds to metallic drops. The small segment in the upper curve close to the full line is the bubble state. The rest of the dashed curves correspond to stripes. The full line is the uniform metal.

Inside the FPS-state one can see that the high density "geometrical" transition from the "bubble" state to the striped one for $\lambda<\lambda^{\star}$ and the low density "geometrical" transition from the latter state to the metal drop state have a discontinuity of both the volume fraction and the B local density [Figs. (5), (6)] reflecting the first-order nature of the transitions. This is expected since at least in our approach one can not continuously deform drops to get stripes.

For all the FPS-states, increasing the strength of the long-range interaction the metallic density decreases (Fig. 5) in order to minimize the mixing energy of the inhomogeneous state which is $\propto \lambda n_{B}$. At the same time, increasing $\lambda$ (Fig. 6), the volume fraction has a growing rate larger than in the ordinary $\mathrm{MC}$ case.

The order of the transitions can be also checked if one looks to the chemical potential $\mu=\partial f / \partial n$ (Fig. [7). In the range of stability of the FPS "bubble" state ( small $\lambda$ ) the chemical potential at the critical density has a cusp indicating the second-order nature of the transition. Increasing $\lambda$ to values greater than $\lambda^{\star}$ the "bubble" state disappears and the chemical potential has a discontinuity at the transition to the striped FPS-state. A similar discontinuity in $\mu$ is also obtained in correspondence to the "geometrical" transitions consistent with the fact that these transitions are first-order like. Notice that for the insulator, which is incompressible, the chemical potential is not defined.

Finally we analyze the size $R_{d}$ of the inhomogeneities and the size $R_{c}$ of the cells in which the system is divided. One can introduce a length scale $l_{d}$ which fixes the natural units for the characteristic size of the cell. This scale correspond to $R_{c}$ (Eqs. (6), (7)) evaluated at 

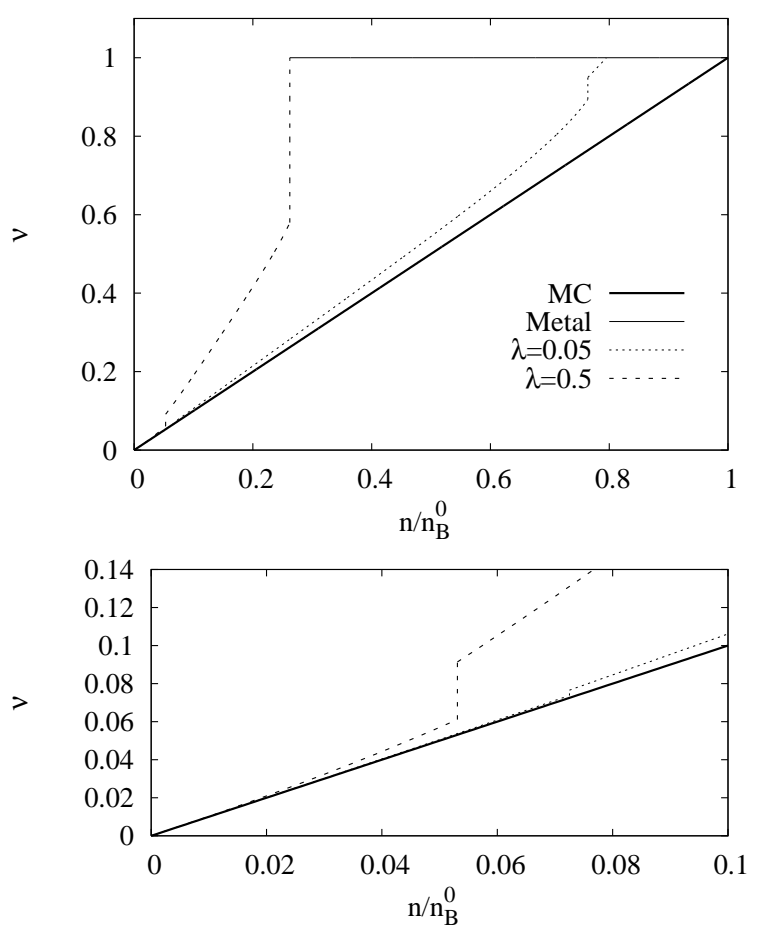

FIG. 6: Top: The volume fraction of the B-metallic phase in the FPS-state vs $n$ at different values of $\lambda$ compared with the ordinary Maxwell construction analysis. The full horizontal line corresponds to the uniform metal. For $\lambda=0.05$ the small segment close to the uniform metal is the bubble state. Close to the origin for both values of $\lambda$ one has the drop state. The rest is in the stripe state. Bottom: Expanded scale at low density to show the abrupt change of $\nu$ due to the "geometrical" transition from the metal drop state to the striped state as density increases.



FIG. 7: The chemical potential behavior in the FPS-state. When the "bubble" state exists, at the transition from the metal to the FPS-state $\mu$ exhibits a cusp point $\left(2^{n d}\right.$ order transition) while for $\lambda>\lambda^{\star}$ the transition to the striped state implies a chemical potential discontinuity $\left(1^{\text {st }}\right.$ order transition)

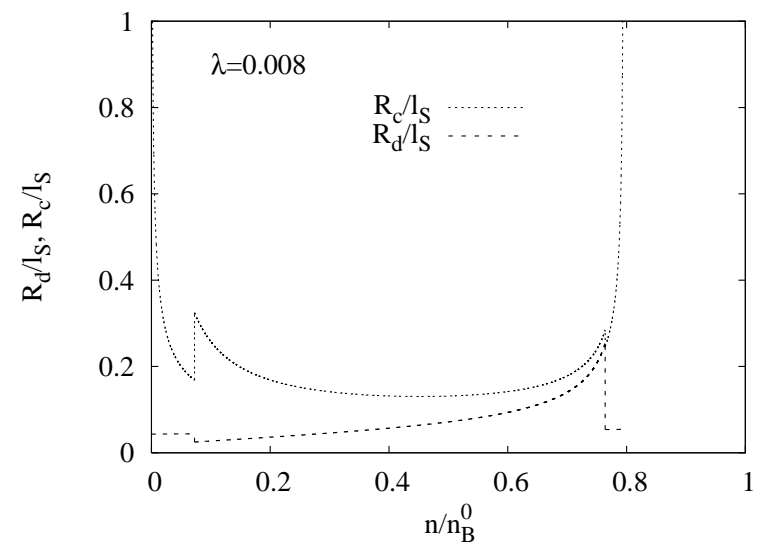

FIG. 8: The cell radius and the inhomogeneity radius vs the global density for small $\lambda$. The divergence in $R_{c}$ are in correspondence to the transitions from the FPS-state to both the metal and the insulator.

the MC-density $n_{B}^{0}$ dropping all the geometric factors:

$$
l_{d}=\left(\frac{\sigma \varepsilon_{0}}{e^{2}\left(n_{B}^{0}\right)^{2}}\right)^{\frac{1}{2}}
$$

Another important length scale is the screening length:

$$
l_{s}=\frac{\varepsilon_{0}}{2 \pi e^{2} \kappa_{B}}
$$

Inserting the last equation in the definition of $\lambda$ one finds that $\lambda=l_{d} /\left(2 \pi l_{s}\right)$. Similar length scales where defined in the $3 \mathrm{D}$ case, 34 in particular we can write the screening length for general dimension (neglecting factors of $\pi$ ) $l_{s} \sim\left(\varepsilon_{0} /\left(e^{2} \kappa_{B}\right)\right)^{1 /(d-1)}$. In $3 D$ systems $l_{S}$ gives an upper limit for the size of inhomogeneities $\stackrel{34.35 .36}{\underline{3}}$ The nature of screening is quite different in $2 \mathrm{D}$ systems ${ }^{49}$ As it will be clear in Sec. IV $l_{s}$ still plays a fundamental but does not limit the size of inhomogeneities.

With the above definitions one has:

$$
\begin{gathered}
R_{c}=2 \pi \lambda l_{s} \frac{1}{n_{B}^{\prime}} \frac{2}{u(\nu)} \quad \text { Stripes } \\
R_{c}=2 \pi \lambda l_{s} \frac{1}{n_{B}^{\prime}} \frac{4 \sqrt{\nu(1-\nu)}}{u(\nu)} \quad \text { Drops }
\end{gathered}
$$

For the stripes the half-width of the metallic regions is given by $R_{d}=R_{c} \nu$. For the drop geometry it is more convenient to define a symmetrized radius $R_{d}=R_{c}[\nu(1-$ $\nu)]^{\frac{1}{2}}$ that smoothly interpolates between the radius of metallic drops at small $\nu$ and the radius of bubbles for $\nu$ close to 1 . The above assumptions lead to the simple expression,

$$
R_{d}=\sqrt{3} \pi \frac{\lambda l_{s}}{\sqrt{1-\frac{16}{\sqrt{3}} \lambda n^{\prime}}}=\frac{\sqrt{3}}{2} \frac{l_{d}}{\sqrt{1-\frac{16}{\sqrt{3}} \lambda n^{\prime}}} .
$$


For small $n^{\prime}$ or small $\lambda$ one has an almost constant behavior $R_{d} \sim 0.9 l_{d}$ [c.f. Figs. 889].

For $\lambda<\lambda^{*}$ bubbles appear in the metal with a divergence of the cell radius while the drop radius remains finite (see Fig. 8). That is at threshold bubbles appear suddenly with a finite size but the transition is second order because they are infinitely far apart. This is reminiscent of the transition in a type II superconductor as a function of field at $H_{c_{1}}$, which according to GL theory is second-order although normal state "drops" (the vortex core) have a finite radius $\xi \underline{\underline{50}}$

For $\lambda<\lambda^{\star}, R_{d}$ is of the order of $l_{s}$. If the latter is of the order of the interparticle distance, a mesoscopic treatment may be problematic. On the other hand increasing $\lambda, R_{d}$ grows to values that are much larger than $l_{s}($ Fig Q). This represents the main difference respect to $3 \mathrm{D}$ systems for which it was demonstrated that $l_{s}$ is an upper bound for $R_{d}$ at any $\lambda$. In the next section we will show that this difference stands on the different role of the LRC interaction and screening in 2D and 3D systems.

The divergence of the cell radius disappear when $\lambda$ is greater than $\lambda^{\star}$, since at the first-order transition to the striped state the cell radius stays finite.

Increasing $\lambda$ at fixed global density one finds that $R_{c} / l_{s}$ increases. This behavior is easy to rationalize if one considers an increase of the surface energy in such a way that $\lambda$ increases while keeping $l_{s}$ constant. In this case the system prefers to make domains with longer periodicity to reduce the surface energy. From Fig. 9 we see that metallic stripes become narrower as the insulator is approached.

\section{LOCAL DENSITY APPROXIMATION}

In this section we want to relax the uniform density approximation. For simplicity as in Sec. III] we restrict ourselves to study a phase separation between a metallic phase $B$ and an insulating phase $A$ with energy density $f_{A}=0$.

In order to account for the spatial dependence of the local density, we take the total free energy as a local density functional. Indicating with $V_{B}$ the B-phase domain volume and with $V$ the total system volume the total free energy can be put as:

$$
\begin{aligned}
F= & \int_{\boldsymbol{r} \in B} f_{B}\left(n_{B}(\boldsymbol{r})\right) d^{2} r+\sigma \Sigma_{A B}+\frac{1}{2 \varepsilon_{0}} \iint \frac{e^{2}}{\left|\boldsymbol{r}_{1}-\boldsymbol{r}_{2}\right|} \\
& {\left[n_{B}\left(\boldsymbol{r}_{1}\right)-\bar{n}\right]\left[n_{B}\left(\boldsymbol{r}_{2}\right)-\bar{n}\right] d^{2} r_{1} d^{2} r_{2} }
\end{aligned}
$$

where $\bar{n}$ is the global density, while $\Sigma_{A B}$ is the total interface surface. We still assume a sharp interface due to short range forces with the parameter $\sigma$ parameterizing the surface energy. Eventually the surface energy term could be replaced by a gradient term to consider soft interfaces.

The constraint of charge neutrality of the system reads:
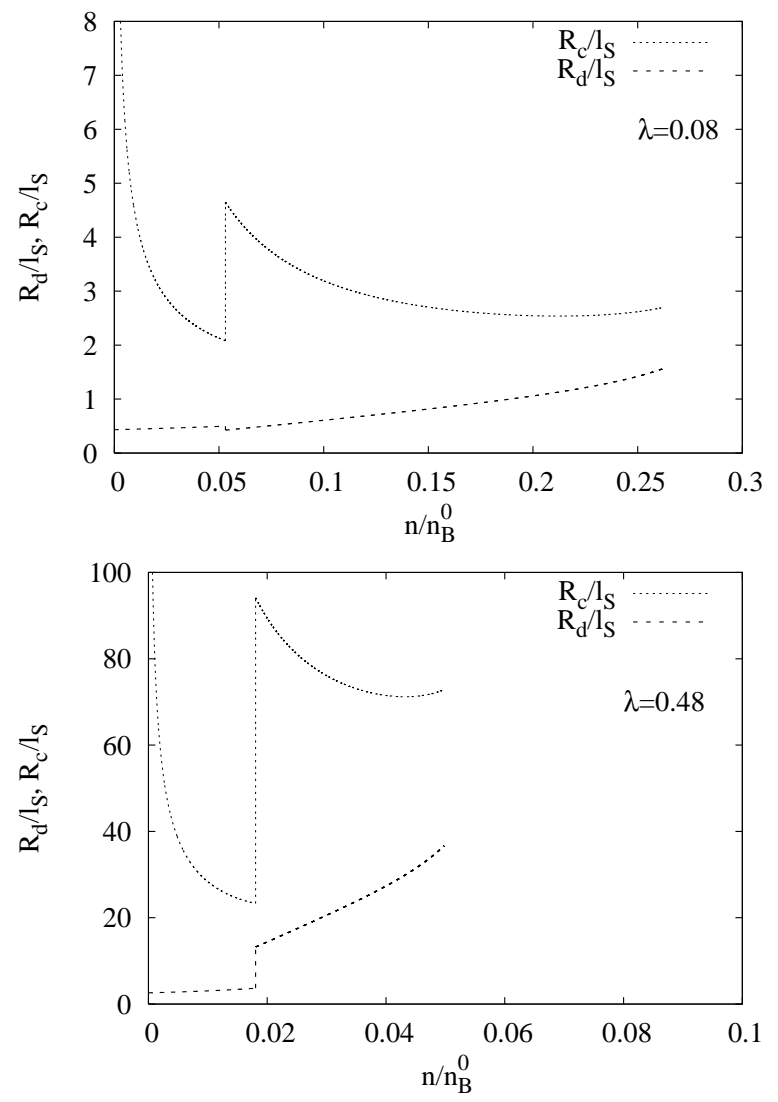

FIG. 9: Top: $R_{c}$ and $R_{d}$ vs. $n$ for $\lambda=0.08$. The FPS-state appear with a finite cell radius. The typical size of the inhomogeneities is of the order of $l_{S}$. Bottom: Same of top for $\lambda=0.48$. The sizes of the domains and the cells are much greater than $l_{S}$.

$$
\int_{\boldsymbol{r} \in B} n_{B}(\boldsymbol{r}) d^{2} r=\bar{n} V
$$

We minimize the total free energy functional with respect to the local density and include the above neutrality constraint via a Lagrange multiplier $\mu_{e}$. We then obtain an equation which tell us that the electrochemical potential is constant:

$$
\mu_{B}(\boldsymbol{r})-\frac{e}{\varepsilon_{0}} \phi(\boldsymbol{r})=\mu_{e} \quad \forall \boldsymbol{r} \epsilon B
$$

where $\phi(\boldsymbol{r})$ indicates the electrostatic potential generated by the charge distribution $\left[n_{B}(\boldsymbol{r})-\bar{n}\right]$. This equation has to be solved together with the neutrality condition Eq. (26).

Assuming again for the B-phase the parabolic free energy [Eq. [16] ], one obtains an equation relating the local density to the potential in terms of the compressibility:

$$
n_{B}(\boldsymbol{r})-n_{B}^{0}=\kappa_{B}\left[\frac{e}{\varepsilon_{0}} \phi(\boldsymbol{r})-\left(\mu_{0}-\mu_{e}\right)\right] \quad \forall \boldsymbol{r} \epsilon B
$$




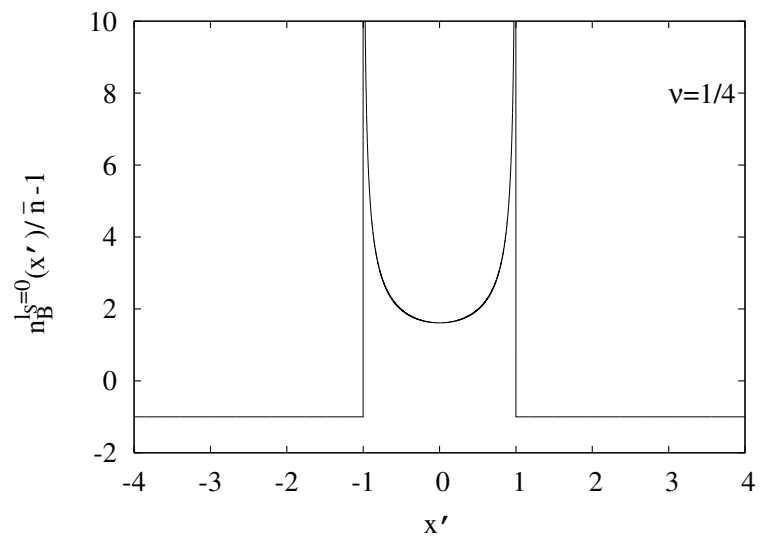

FIG. 10: The excess charge density profile $n_{B}^{l_{s}=0}\left(x^{\prime}\right) / \bar{n}-1$ in the direction perpendicular to the stripe for $\nu=1 / 4$. $x^{\prime}$ is the component of $\boldsymbol{r} / R_{d}$

or in terms of the screening length

$$
\phi(\boldsymbol{r})-\frac{\varepsilon_{0}}{e}\left(\mu_{0}-\mu_{e}\right)=2 \pi e l_{s}\left[n_{B}(\boldsymbol{r})-n_{B}^{0}\right] \quad \forall \boldsymbol{r} \in B
$$

In the limit of infinite compressibility i.e. zero screening length, the electrostatic potential is constant on the metallic regions and therefore $n_{B}(\boldsymbol{r})$ correspond to the distribution of a metal for which the 3D Laplace equation $\nabla^{2} \phi=0$ is supplemented by the boundary condition $\phi=$ const on the domains. Here we are assuming that $l_{s} / R_{d} \rightarrow 0$ but we are not making assumptions about the other parameters so that $\lambda \sim l_{d} / l_{s}$ may remain finite.

In this limit the problem can be solved analytically for the stripes geometry. In fact, in this case the Coulomb potential is calculated by using the Schwarz-Christoffel conformal transformations. 51 The B-phase density spatial dependence comes out to be:

$$
n_{B}^{l_{S}=0}\left(x^{\prime}\right)=\bar{n} \frac{\left|\cos \frac{\pi x^{\prime} \nu}{2}\right|}{\sqrt{\sin ^{2} \frac{\pi \nu}{2}-\sin ^{2} \frac{\pi x^{\prime} \nu}{2}}}
$$

where $x^{\prime}$ indicates the $x$ component of the dimensionless coordinate $\boldsymbol{r}^{\prime}$ defined by $\boldsymbol{r}^{\prime}=\boldsymbol{r} / R_{d}$. In Fig. (10) we show the spatial dependence of the excess charge density $n_{B}^{l_{s}=0}\left(x^{\prime}\right) / \bar{n}-1$ on one unit cell.

Keeping in mind that the charge in the stripe region is undercompensated by the background, one finds that at this level of approximation the metallic stripe behaves as a macroscopic charged metallic strip. The density accumulates on the border of the stripe and decays as a power law towards the center.

It is interesting to compare this configuration with the three dimensional analogue, that is metallic layers locally undercompensated by a uniform background. In that case the charge is localized at the surface of the layer and decays as $\exp \left(-x / l_{s}\right)$. Although in both cases $l_{s}$ is the length scale below which macroscopic electrostatic, i.e $\phi=$ const., is not any more valid, the solutions for $x>>l_{s}$ are dramatically different in the two cases. For $3 \mathrm{D}$ metals the local density far from the surface is forced to be equal to the background density which in turn is equal to the global electronic density i.e. $n_{B}=\bar{n} .34$ On the contrary on the present $2 \mathrm{D}$ case the local density far from the surface can be quite different from the global density.

In Ref. 34,36 it was shown that for small $l_{S} / l_{d}$ in the $3 \mathrm{D}$ case mesoscopic inhomogeneous phase separation is forbidden. In fact the system gains phase-separation energy when the local density differs from the global density value. Instead electrostatic forces $n_{B}=\bar{n}$, except for the microscopic length $l_{S}$, and the system remains uniform. In the $2 \mathrm{D}$ case the inhomogeneity is able to gain phase separation energy in all the region where $n_{B} \neq \bar{n}$ which is not limited by a microscopic length.

A related issue in $3 \mathrm{D}$ is that in general inhomogeneities can not have all linear dimensions larger than $l_{s}$. In the $2 \mathrm{D}$ case this does not impose any constraint because the linear size perpendicular to the layer is from the outset smaller than $l_{s}^{3 D}$. Therefore inhomogeneities can have an unbound size in the plane.

Notice that this different behavior between 2D and 3D systems is due to the difference in the charge profile far from the surface. Of course the divergence of the electronic density at the surface of the stripe is unphysical and will be cutoff by a microscopic length (see below) but this does not affect the behavior of the charge far from the surface that is essential for our argument.

In the related problem of a two-dimensional electron (hole) gas in MOSFET devices the background is not rigid but is provided by a metallic gate in the form of mobile holes (electrons). In this case the background can relax and other considerations enter into play 39.40 In the case in which the separation between the gate and the e-gas is much larger than $R_{c}$ and $R_{d}$, the electric field produced by the electron gas inhomogeneities will be perpendicular to the gate plane and this will prevent the background to relax. This is illustrated in Fig. 11 where we show the equipotential lines in the plane perpendicular to the stripe. We see that at a distance $\sim R_{c}$ from the stripe the equipotential lines become parallel to the stripe surface. In this case the background behaves as rigid and our results apply. In other words the background can follow the inhomogeneities of PS only when the inhomogeneities are comparable in size to the separation between the e-gas and the gate. The crossover between these two possible scenarios will be considered elsewhere. ${ }^{41}$.

To further clarify the role of $l_{S}$, now we calculate the charge density profile for $l_{S} \neq 0$ adapting the method of Ref. 49. The unphysical divergence of the metallic density will be removed. We restrict to the stripe geometry as above but the same method can be used for other geometries. Consider the 3D Poisson equation

$$
\nabla^{2} \phi^{3 D}(\boldsymbol{r}, z)=-4 \pi \rho(\boldsymbol{r}, z)
$$




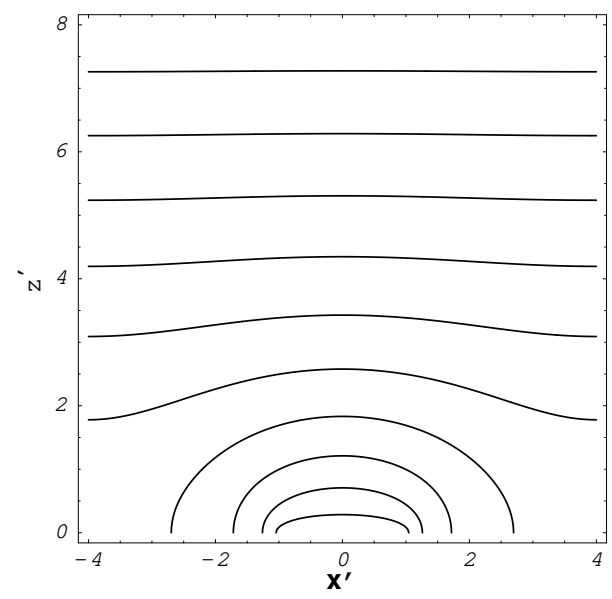

FIG. 11: The equipotential lines in the plane perpendicular to the stripes for the same parameters as Fig. 10

with $z$ the distance from the plane and the 3D charge density defined as $\rho(\boldsymbol{r}, z)=\rho(\boldsymbol{r}) \delta(z)$ with $\rho(\boldsymbol{r})$ the 2D in plane density. To solve Eq. (31), we perform the Fourier transform in the $x$ direction using the fact that the solution is periodic in $2 R_{c}$ :

$$
\begin{aligned}
& \phi^{3 D}(x, z)=\frac{1}{2 R_{c}} \sum_{q} e^{i q x} \phi^{3 D}(q, z) \quad q=\frac{\pi n}{R_{c}} \\
& \phi^{3 D}(q, z)=\int_{-R_{c}}^{R_{c}} d x e^{-i q x} \phi^{3 D}(x, z)
\end{aligned}
$$

(and similarly for the charge distribution). Since there is no dependence in the direction of the stripe we dropped the $y$ coordinate.The Poisson equation is then:

$$
\frac{\partial^{2} \phi^{3 D}(q, z)}{\partial z^{2}}-q^{2} \phi^{3 D}(q, z)=-4 \pi \rho(q) \delta(z)
$$

with $\rho(q)$ defined as the Fourier transform of the in plane density

$$
\rho(\boldsymbol{r}) \equiv-e\left[n_{B}(\boldsymbol{r})-\bar{n}\right]
$$

For $z \neq 0$ the solution for the potential is $\phi^{3 D}(q, z)=$ $\phi^{3 D}(q, 0) e^{-|q||z|}$. Integrating Eq. (33) in a small interval one obtains the boundary condition:

$$
|q| \phi(q)=2 \pi \rho(q)
$$

with the Fourier transform of the in-plane electrostatic Coulomb potential defined as $\phi(q) \equiv \phi^{3 D}(q, 0)$. The 3D boundary condition Eq. (34) looks formally like an effective 2D Poisson equation.

Eq. (28) or Eq. (29) determines how the charge responds to the potential and Eq. (34) determines how the potential is generated by the charges. Both equations must be solved self-consistently in order to find the charge distribution.

Using the superposition principle both charge and potential can be written as the sum of the terms evaluated above for infinite compressibility (i.e. for $l_{s}=0$ ) plus a correction, which we wish to compute:

$$
\begin{aligned}
\phi(\boldsymbol{r}) & =\phi^{l_{S}=0}(\boldsymbol{r})+\delta \phi(\boldsymbol{r}) \\
n_{B}(\boldsymbol{r}) & =n_{B}^{l_{S}=0}(\boldsymbol{r})+\delta n_{B}(\boldsymbol{r})
\end{aligned}
$$

The correction $\delta \phi(\boldsymbol{r})$ satisfy the effective Poisson equation:

$$
|q| \delta \phi(q)=-2 \pi e \delta n_{B}(q)
$$

The unknown Lagrange multiplier $\mu_{e}$ has to be determined by fulfilling the neutrality condition and can also change as $l_{s}$ is increased from zero:

$$
\mu_{e}=\mu_{e}^{l_{s}=0}+\delta \mu_{e}
$$

Eq. (29) can be put as

$$
\begin{aligned}
\delta n_{B}(\boldsymbol{r})= & \frac{1}{2 \pi e l_{S}} \delta \phi(\boldsymbol{r})-n_{B}^{l_{S}=0}(\boldsymbol{r})+n_{e} \\
& \forall \boldsymbol{r} \in B
\end{aligned}
$$

where we have absorbed the Lagrange parameter in the constant $n_{e}=n_{B}^{0}+\frac{\varepsilon_{0}}{2 \pi e^{2} l_{s}} \delta \mu_{e}$. In Fourier space we get:

$$
\begin{aligned}
\delta n_{B}(q) & =\frac{1}{2 \pi e l_{S} R_{c}} \sum_{q^{\prime}} \delta \phi\left(q^{\prime}\right) \frac{\sin \left[\left(q-q^{\prime}\right) R_{d}\right]}{\left(q-q^{\prime}\right)} \\
& -n_{B}^{l_{S}=0}(q)+n_{e} \frac{2 \sin \left(q R_{d}\right)}{q} .
\end{aligned}
$$

Eq. (37) and Eq.(39) are a closed system since the quantities with $l_{S}=0$ are known from the previous treatment.

In the case $R_{c}, R_{d}>>l_{S}$ one can substitute $\sum_{q} \rightarrow$ $2 R_{c} \int d q /(2 \pi)$ and make the approximation:

$$
\frac{2 \sin \left[\left(q-q^{\prime}\right) R_{d}\right]}{\left(q-q^{\prime}\right)} \rightarrow 2 \pi \delta\left(q-q^{\prime}\right) .
$$

Using Eq. (37) we obtain:

$$
\delta n_{B}(q)=\frac{2 n_{e} \sin \left(q R_{d}\right) / q-n_{B}^{l_{S}=0}(q)}{1+1 /\left(l_{S}|q|\right)}
$$

In the limit $q \rightarrow 0$ we obtain $\delta n_{B} \rightarrow 0$. The uniform component of the charge does not change and therefore $\delta \mu_{e}=0$ and $n_{e}=n_{0}^{B}$. We have evaluated the above expression via a discrete Fourier transform in the limit in which the stripes are far apart $\left(R_{c} / R_{d} \rightarrow \infty\right.$ i.e $\left.\nu \rightarrow 0\right)$. This correspond to solve the problem for a single stripe. The electronic density at $l_{S}=0$ can be put as:

$$
n_{B}^{l_{S}=0}\left(x^{\prime}\right)=\frac{2 \bar{n}}{\pi \nu} \frac{1}{\sqrt{1-\left(x^{\prime}\right)^{2}}}
$$

In Fig. 12 we show the total electronic charge density for $l_{S} / R_{d}=0.03$. The main difference with respect to 

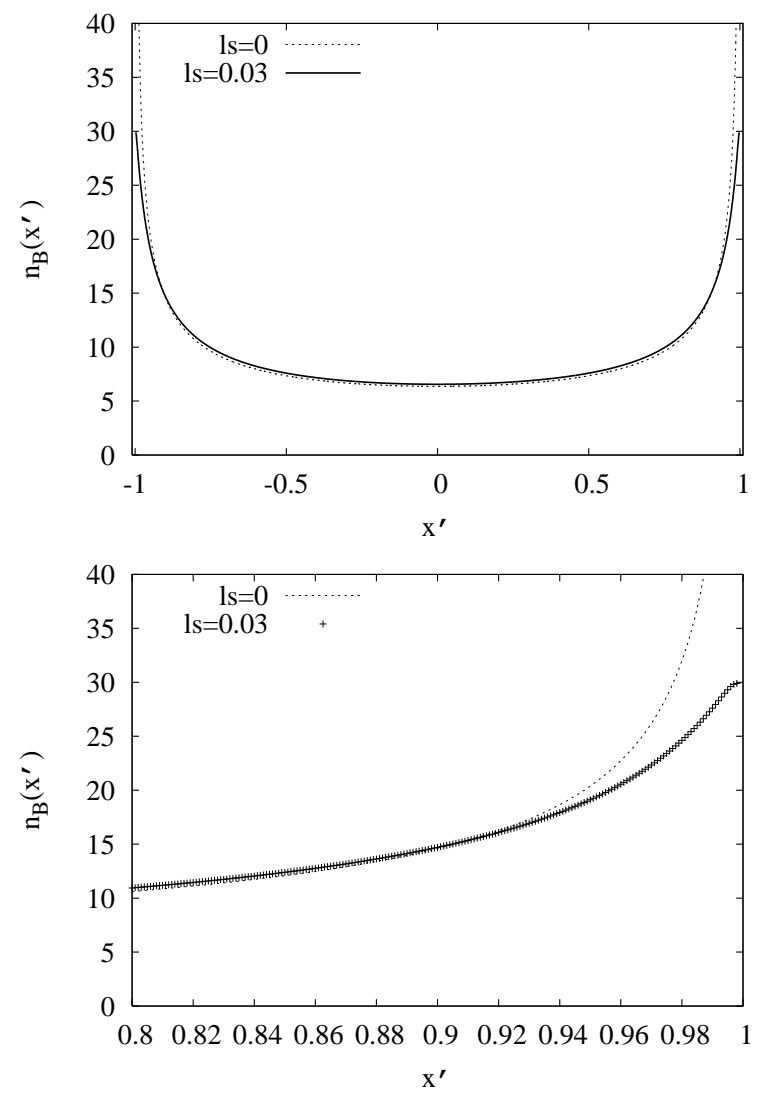

FIG. 12: Top panel: Comparison between the electronic charge density at $l_{S} / R_{d}=0$ and $l_{S} / R_{d}=0.03$ evaluated at $\nu=1$ and $\bar{n}=1$. Bottom panel:Expansion near the inhomogeneity surface

the $l_{S}=0$ case is that the unphysical divergence of the density at the stripe surface is removed and the density tends to a finite value at the stripe boundary. Notice that at distances from the interface larger than $l_{S}$ the charge density at $l_{S} \neq 0$ practically coincides with the one at $l_{S}=0$ as expected. Our previous conclusion regarding the absence of upper bounds for the size of the domains remains unchanged. In the entire domain the local density differs from the average density and therefore the phase separation energy gain comes from the whole domain and not from the electric field penetration depth as it happened in the 3D case.

Finally one has that the electronic potential is given by:

$$
\delta \phi_{e}^{3 D}(q)=-\frac{2 \pi e}{|q|+\frac{1}{l_{S}}}\left[n_{e} \frac{2 \sin \left(q R_{d}\right)}{q}-n_{B}^{l_{S}=0}(q)\right]
$$

The above electrostatic potential includes the twodimensional screening with $l_{S}$ as a screening parameter. It is of the same form of the screened potential discussed in Ref. 49 .

\section{DISCUSSION AND CONCLUSION}

In this work we have considered the problem of mesoscopic FPS in 2D electronic systems frustrated by the LRC interaction and the surface energy. In particular we concentrated on the problem of coexistence between a $2 \mathrm{D}$ metal and an insulator.

We have supposed that the inhomogeneous state is realized with two different geometric arrangements: disks of one phase into the other and a state with alternating stripes of metal and insulator. The first arrangement comes in two different flavors: bubbles of insulator hosted by the metal for $\nu \sim 1$ and metal drops hosted by the insulator for $\nu \sim 0$.

We have defined a parameter $\lambda$ which specifies the strength of the frustration and is given by the ratio of the characteristic mixing energy to the characteristic phase separation energy gain in the absence of frustration.

As in the 3D case, we have found that frustration tends to extend the global density region where the uniform metallic phase is stable. Within our approximation, below some critical value of $\lambda$, we have found that as the global density is reduced there is a second order transition from the homogeneous metallic state to an inhomogeneous state with insulating bubbles in the metal. Above this value of $\lambda$ the bubble state is never stable and the inhomogeneous transition leads to the striped state with a first-order transition. Inside the inhomogeneous stability region by decreasing the global density first-order "topological" transitions induces changes in the geometry of the domains.

In Sec. IIIA 1 we have analyzed the inhomogeneous state properties and we have found that the size of inhomogeneities is not forced to have one linear dimension smaller than the screening length $l_{s}$ in sharp contrast with the $3 \mathrm{D}$ case. This difference stands on the qualitative different behavior of the screening in a $3 \mathrm{D}$ and in a $2 \mathrm{D}$ system. In the first case the screening decays exponentially from the interfaces whereas in the second case it decays as a power law. In this last case it is possible to gain PS energy from regions far from the skin depth.

Inhomogeneities seem to be related to a number of interesting phenomena like colossal magnetoresistent. Our study suggests that inhomogeneities may be favored by engineering materials with enhanced anisotropy. For example adding insulating layers in between metallic layers should favor mesoscopic phase separation.

A realistic model of the 2DEG would require considering a metallic phase with negative compressibility as observed in the uniform phase. The effects of disorder should also be included. ${ }^{41}$ Therefore here we limit our discussion to some generic qualitative features. The stabilization effect of the long-range Coulomb interaction has been observed on the 2DEG where the state with negative short-range electronic compressibility has been show to be stable in a certain range of densities 9.10 .25 .26 .27 .28 Ilani and collaborators $\$ 9.10$ observed that the system becomes inhomogeneous at a mesoscopic scale when the 
system is at the verge of the metal-insulator transition suggesting a connection between the two phenomena.

Another interesting finding of Ilani and collaborators 9.10 is that coming from the metallic side the compressibility has negative spikes close to the transition. When integrated those spikes imply a decreasing step in the chemical potential as a function of density. This behavior is similar to what we find for the transition from the inhomogeneous state to the metallic state as the density is increased in the case in which the transition is first order (c.f. Fig. 7). The main difference between our result and the experiment is that in our case there is a single large step at the transition whereas Ilani et al. find many small steps around the critical density. This behavior is easy to rationalize if one considers that in the presence of disorder the density will not be uniform in all the sample. A distribution of critical densities and fragmentation of the large step in many small steps will be naturally produced. The minimum step size will be given by the appearance of a single drop.

We have restricted ourselves to one isolated plane but we expect the same physics to be true for weakly coupled planes as found in some cuprates, nickelates manganates and other strongly correlated materials. As mentioned in the introduction the situation in manganites is quite complex due to the large variety of competing phases. In layered cuprates one finds striped charge and spin density waves at small doping and a Fermi liquid at large doping. From the behavior of the mean-field energy ${ }^{52}$ it is quite natural to expect phase separation among the stripe state and the overdoped Fermi liquid. To make a rough estimate of the drop size we identify the stripe state as the pseudogap phase and treat it as incompressible. If we assume that the surface energy is of order $\sigma \sim J / a$, with $J$ the superexchange constant and $a$ the lattice constant, the drop radius can be written as [c.f. Eq. (24)],

$$
R_{d} \sim l_{d} \sim \frac{a}{\Delta x}\left(\frac{\epsilon_{0} a J}{2 a_{0} R y}\right)
$$

where $a_{0}$ is the Borh radius, $\Delta x \sim 0.1$ is the range of doping where one expects phase separation according to MC. Using $J=0.01 R y, a=7.2 a_{0}, \epsilon_{0}=5$, one gets $2 R_{d} \sim 8 a \sim 30 \AA$ which compares well with the inhomogeneities found in Ref 13 . This experiments reveals superconducting like inhomogeneities of roughly $50 \AA$ diameter (playing the role of the compressible phase) embedded in insulating like regions. A similar rough estimate gives $\lambda \sim 1$.

The constraint on the maximum size of inhomogeneities in 3D systems makes the conditions for stability of a phase separated state very stringent 34 In $2 \mathrm{D}$ system instead we have found that this constraint does not apply and frustrated phase separation at the mesoscopic scale is much more favorable. This may be the reason why inhomogeneities are often found in quasi two-dimensional electronic systems.

\section{Acknowledgments}

C. Ortix acknowledges partial support from INFM and Dipartimento di Fisica, Università di Roma "La Sapienza" during part of this work and M. Beccaria for useful discussions.

\section{APPENDIX A: ELECTROSTATIC ENERGY IN THE UNIFORM DENSITY APPROXIMATION}

Here we compute the explicit expressions of the electrostatic energy within the UDA for the two geometries considered. We start by considering regular arrays of inhomogeneities and dividing the system in Wigner-Seitz cells in such a way that each cell is globally neutral. The electrostatic energy is $N_{d}$ times the Coulomb energy of one cell.

As in Ref. 34 we take the density profile inside each cell as $-e\left(n_{B}-n_{A}\right)$ inside the B-phase inhomogeneity of radius $R_{d}$ compensated by the background charge density $e\left(n-n_{A}\right)$.

In $3 \mathrm{D}$ the computation of the Coulomb energy is facilitated by the use of Gauss theorem ${ }^{34}$ In the present 2D case with the full 3D Coulomb interaction Gauss theorem is not useful so we use the explicit expression: $:^{53}$

$$
e_{e l}=\frac{1}{2 \varepsilon_{0}} \frac{1}{V_{d}} \int_{V_{d}} d^{2} \boldsymbol{r}\left(\rho_{d}+\rho_{b}\right)\left(\phi_{d}(\boldsymbol{r})+\phi_{b}(\boldsymbol{r})\right)
$$

where $V_{d}$ is the $2 \mathrm{D}$ volume of one cell, $\rho_{d} \equiv$ $-e\left(n_{B}-n_{A}\right)$ is the inhomogeneity charge density and $\rho_{b}=e\left(n-n_{A}\right)$ is the effective background charge density. We need to evaluate the Coulomb potential $\phi_{d}$ generated by the inhomogeneities of all cells and the Coulomb potential $\phi_{b}$ generated by the whole background charge density. The latter corresponds to the Coulomb potential of an infinite uniformly charged plane and can be taken as constant in the plane. Because of the global neutrality the value of the constant does not affect the electrostatic energy and we can take $\phi_{b}=0$.

The inhomogeneity Coulomb potential is the sum of the $N_{d}$ contributions from each cell. We separate the contribution of the cell where we are integrating from the contribution of the other cells. In this way Eq. (A1) separates in a self-energy contribution $\left(e_{e l}^{\Sigma}\right)$ and an interaction contribution $e_{e l}^{i n t}$ :

$$
\begin{aligned}
e_{e l}^{\Sigma} & =\frac{V_{d}^{-1}}{2 \varepsilon_{0}} \int_{V_{d}} d^{2} \boldsymbol{r}\left(\rho_{d}+\rho_{b}\right) \phi^{\Sigma}(\boldsymbol{r}) \\
e_{e l}^{i n t} & =\frac{V_{d}^{-1}}{2 \varepsilon_{0}} \int_{V_{d}} d^{2} \boldsymbol{r}\left(\rho_{d}+\rho_{b}\right) \phi^{i n t}(\boldsymbol{r})
\end{aligned}
$$

\section{Stripe geometry}

In this geometry we assume that the system is divided in cells of width $2 R_{c}$ and length $L$ with periodic bound- 
ary conditions. Within each cell the width of the inhomogeneity with charge density $\rho_{d}$ is equal to $2 R_{d}$.

To compute the electrostatic energy we have evaluated the two expressions Eqs. (A2), A3 . The interaction potential $\phi^{i n t}$ has been numerically computed truncating the sum of the contribution from each cell to a finite number of cells: $N_{c}$. For $N_{c}$ not too large the Coulomb energy is asymmetric. However for $N_{c}$ of the order of $10^{2}$ the Coulomb potential becomes symmetric respect to the "phase-exchange" symmetry $(A \rightarrow B, \nu \rightarrow 1-\nu)$ indicating the achieved convergence.

In the limit $\nu \rightarrow 0, R_{d}<<R_{c}$, $\phi^{\text {int }}$ can be neglected so that the total electrostatic energy is well approximated in this limit by:

$$
\lim _{\nu \rightarrow 0} e_{e l}^{\Sigma}=\frac{e^{2}}{\varepsilon_{0}}\left(n_{B}-n_{A}\right)^{2} R_{c} 2 \nu^{2}[-\ln \nu]
$$

We can obtain a similar approximation in the opposite limit $\nu \rightarrow 1$ by imposing the "phase-exchange" symmetry. This leads to the approximate expression Eq. (5) which interpolates between the $\nu \rightarrow 0,1$ limits. The comparison between the approximate and the numerical result is shown in Fig. 1] in which are reported the $u$ functions considering the different electrostatic energies. We see that the approximation to keep only the self energy term is indeed very good.

\section{Drop geometry}

From the computation in the stripe geometry it is clear that in the limit of $\nu \rightarrow 0$ and $\nu \rightarrow 1$ the dominant electrostatic term is the self-energy. Since the drops are zero dimensional objects we expect that the effect of the intercell terms to be even smaller than for the case of stripes. To check this hypothesis we first estimate the interaction energy between the cells in this way: if we look to the total system of $N_{d}$ cells in the limit of small volume fraction, the drops can be considered as negative point-charges at distance $R_{c}$ since $\nu \rightarrow 0$ is equivalent to $R_{d}<<R_{c}$ and we assume that the drops arrange in a Wigner crystal. The electrostatic energy is given by: ${ }^{54}$

$$
E_{e l}^{i n t} \propto-\alpha \frac{\left[e R_{d}^{2}\left(n_{B}-n_{A}\right)\right]^{2}}{R_{c}}
$$

Since $\nu=R_{d}^{2} / R_{c}^{2}$ for the drop geometry and referring to the energy per unit volume one has:

$$
e_{e l}^{i n t} \propto \frac{e^{2}}{\varepsilon_{0}}\left(n_{B}-n_{A}\right)^{2} R_{c} \alpha \nu^{2}
$$

The Coulomb self-energy of a cell of radius $R_{d}$ can be evaluated noting that the cell charge density in units of $(-e)$ can be written in the Fourier space as ${ }^{55}$ :

$$
\delta n(k)=2 \pi\left(n_{B}-n_{A}\right) R_{d}^{2}\left(\frac{J_{1}\left(k R_{d}\right)}{k R_{d}}-\frac{J_{1}\left(k R_{c}\right)}{k R_{c}}\right)
$$

where $\delta n(k)=\left[n_{B}(k)-n_{A}(k)\right]$. The electrostatic energy per unit volume is:

$$
e_{e l}^{\Sigma}=\frac{e^{2}}{2 \varepsilon_{0} \pi R_{c}^{2}} \int \frac{d^{2} k}{(2 \pi)^{2}}\left(n_{B}(k)-n_{A}(k)\right)^{2} \frac{2 \pi}{k}
$$

Computing the coulombic self-energy per unit volume in the Fourier-space one obtains:

$$
e_{e l}^{\Sigma}=\frac{e^{2}}{\varepsilon_{0}}\left(n_{B}-n_{A}\right)^{2} R_{c} \frac{8}{3}\left[\nu^{\frac{3}{2}}+o\left(\nu^{2}\right)\right]
$$

were we have kept the dominant contribution when $\nu \rightarrow$ 0 . Also for drops one obtains that in this limit the selfenergy term dominates the electrostatic energy.

The limit $\nu \rightarrow 1$ can be obtained by replacing $\nu \rightarrow$ $1-\nu$. This leads to the approximate electrostatic energy expression Eq. (4) which as for stripes interpolates between the two limits.

Notice that since the inter drop interaction is negligible our computation is independent of the lattice structure of the crystal and is also valid for an amorphous configurations of drops.

\section{APPENDIX B: LIMIT OF SMALL SCREENING LENGTH IN THE UDA AND COMPARISON BETWEEN THE UDA AND THE LDA}

To compare the LDA and the UDA we use the particularly simple limit $l_{S} \rightarrow 0$. Formally this can be achieved by making $\kappa_{B} \rightarrow \infty$ so that the $B$ phase bulk energy becomes a linear function of density. If $n_{B}^{0}$ is kept constant the line intersects the energy of the A-phase in this limit.

We will instead consider the energy density difference $\Delta E_{g}$ between the $B$ and the $A$ phase at zero density fixed. In this case the $\mathrm{MC}$ density $n_{B}^{0}=\left[\Delta E_{g} \kappa_{B}\right]^{1 / 2}$ diverges.

To make things less abstract we can consider the following example: classical electrons with a short range attractive interaction in the lattice and at low temperature. The attraction stabilizes a crystal at high density. The energy per unit volume of the uniform crystal is $f_{A}^{0}=-z v n_{0} / 2$ where $z$ is the coordination number $v$ is the short range attraction and $n_{0}$ is the density of the incompressible crystal phase.

For small global density the attraction can be neglected. Electrons form a uniform "metallic" phase. Since the electrons are classical (no tight binding hopping term) the chemical potential becomes independent of the density (thus the compressibility is infinite) and taken to be zero.

In the absence of long-range interaction and for electronic densities $n_{e}<n_{0}$ this system phase separate into the high density crystal phase and the zero density empty phase. In the presence of the long-range Coulomb interaction separation between the high-density crystal phase and a low-density metallic phase becomes possible. 
In order to keep notations consistent with the previous section we consider the hole density $n=n_{0}-n_{e}$. The incompressible $\mathrm{A}$ phase is then at $n=0$ and has energy $f_{A}^{0}<0$ and the infinitely compressible metallic phase has $n>0$ and energy $f_{B}(n)=0$.

The free energies of the homogeneous state and the PS-states (drop and stripes geometries) can be put as:

$$
\begin{aligned}
f_{H}(n) & =0 \\
f_{D, S}(n, \nu) & =(1-\nu) f_{A}^{0}+\left(\frac{\sigma e^{2}}{\varepsilon_{0}}\right)^{\frac{1}{2}} n \frac{u(\nu)}{\nu}
\end{aligned}
$$

Minimizing this expression respect to $\nu$ one obtains the optimum $\nu$ value.

Now in order to test the accuracy of the UDA we will compare this approximation with the LDA derived in Sec. IV For simplicity we restrict to the case of separation between and incompressible phase and an infinitely compressible phase.

The energy can be evaluated in the LDA using the spatial dependence of the electron density found in Sec. IV for $l_{s}=0$ [c.f. Eq. (30)].

As discussed above we assume that $f_{A}$ is a negative constant and

$$
f_{B}\left[n_{B}(\boldsymbol{r})\right]=0
$$

With these conventions the LDA free energy functional reads:

$$
F=V_{A} f_{A}^{0}+\sigma \Sigma_{A B}+\frac{e}{2 \varepsilon_{0}} \int_{V_{A}} \bar{n} \phi^{l_{S}=0}(\boldsymbol{r}) d^{2} \boldsymbol{r}
$$

where we have used that in the metallic phase domains the electrostatic potential for $l_{S}=0$ is constant and thus give no contribution to the Coulomb energy.
For the stripes geometry the energy density reads:

$$
f_{S}=(1-\nu) f_{A}^{0}+\frac{e^{2}}{4 \varepsilon_{0}} \bar{n}^{2} R_{c} \frac{u_{L D A}(\nu)^{2}}{\nu^{2}}+\frac{\sigma}{R_{c}}
$$

Here $u_{L D A}$ is given in terms of the potential at $l_{S}=0$ :

$$
u_{L D A}(\nu)^{2}=\frac{2 \nu^{2}}{e \bar{n} R_{c}} \int_{\nu}^{1} \phi^{l_{S}=0}(\tilde{x}) d \tilde{x}
$$

where $\tilde{x}$ is the dimensionless coordinate $x / R_{c}$ and the potential at $l_{S}=0$ reads:

$$
\phi^{l_{S}=0}(\tilde{x})=4 R_{c} e \bar{n} \cosh ^{-1} \frac{\sin \left|\frac{\pi \tilde{x}}{2}\right|}{\sin \frac{\pi \nu}{2}}
$$

Minimizing the mixing energy $e_{m}=e_{e l}+e_{\sigma}$ respect to the cell radius $R_{c}$ one finally finds:

$$
f_{S}(\bar{n}, \nu)=(1-\nu) f_{A}^{0}+\left(\frac{\sigma e^{2}}{\varepsilon_{0}}\right)^{\frac{1}{2}} \bar{n} \frac{u_{L D A}(\nu)}{\nu}
$$

The difference of energy between the PS and the homogeneous states can be put in the same form as in the UDA Eq. (B2). The only difference with the UDA is encoded in the function $u_{L D A}$. In Fig. (1) we compare the two geometrical $u$ function parameterizing the mixing energies. The LDA function implies a lower mixing energy since we are relaxing the uniform density constraint. This relaxation energy gain however is small demonstrating the accuracy of the UDA approximation for thermodynamic quantities as it was found also in the $3 \mathrm{D}$ case 34
1 in Phase separation in cuprate superconductors, edited by K. A. Muller and G. Benedek (World Scientific, Singapore, 1992).

2 in Phase separation in cuprate superconductors, edited by E. Sigmund and K. A. Muller (Springer-Verlag, Berlin, 1993).

3 A. Moreo, S. Yunoki, and E. Dagotto, Science 283, 2034 (1999).

4 E. L. Nagaev, Physics of magnetic semiconductors (MIR, Moscow, 1983).

${ }^{5}$ E. L. Nagaev, A. I. Podel'shchikov, and V. E. Zil'bewarg, J. Phys.: Condens. Matter 10, 9823 (1998).

${ }^{6}$ M. Hennion, F. Moussa, G. Biotteau, J. RodriguezCarvajal, L. Pinsard, and A. Revcolevschi, Phys. Rev. Lett. 81, 1957 (1998).

7 U. Löw, V. J. Emery, K. Fabricius, and S. A. Kivelson, Phys. Rev. Lett. 72, 1918 (1994).

8 C. Castellani, C. Di Castro, and M. Grilli, Phys. Rev. Lett. 75, 4650 (1995).

9 S. Ilani, A. Yacoby, D. Mahalu, and H. Shtrikman, Phys.
Rev. Lett. 84, 3133 (2000).

10 S. Ilani, A. Yacoby, D. Mahalu, and H. Shtrikman, Science 292, 1354 (2001).

11 S. H. Pan, J. P. O'neal, R. L. Badzey, C. Chamon, H. Ding, J. R. Engelbrecht, Z. Wang, H. Eisaki, S. Uchida, A. K. Gupta, K.-W. Ng, E. W. Hudson, K. M. Lang, and J. C. Davis, Nature (London) 413, 282 (2001).

12 K. McElroy, R. W. Simmonds, J. E. Hoffman, D.-H. Lee, J. Orenstein, H. Eisaki, S. Uchida, and J. C. Davis, nat 422, 592 (2003).

13 K. M. Lang, V. Madhavan, J. E. Hoffman, E. W. Hudson, H. Eisaki, S. Uchida, and J. C. Davis, nat 415, 412 (2002).

14 T. Becker, C. Streng, Y. Luo, V. Moshnyaga, B. Damaschke, N. Shannon, and K. Samwer, Phys. Rev. Lett. 89, 237203 (2002).

15 M. B. Salamon and M. Jaime, Rev. Mod. Phys. 73, 583 (2001).

16 N. M. R. Peres, J. M. P. Carmelo, D. K. Campbell, and A. W. Sandvik, Z. Phys. B 103, 217 (1997).

17 S. Larochelle, A. Mehta, N. Kaneko, P. K. Mang, A. F. 
Panchula, L. Zhou, J. Arthur, and M. Greven, Phys. Rev. Lett. 87, 095502 (2001).

18 L. Zhang, C. Israel, A. Biswas, R. L. Greene, and A. de Lozanne, Science 298, 805 (2002).

19 S. Larochelle, A. Mehta, L. Lu, P. K. Mang, O. P. Vajk, N. Kaneko, J. W. Lynn, L. Zhou, and M. Greven, Phys. Rev. B 71, 024435 (2005).

20 M. Fäth, S. Freisem, A. A. Menovsky, Y. Tomioka, J. Aarts, and J. A. Mydosh, Science 285, 1540 (1999).

21 C. Renner, G. Aeppli, B.-G. Kim, Y.-A. Soh, and S.-W. Cheong, Nature (London) 416, 518 (2002).

22 S.V.Kravchenko, G.V.Kravchenko, J.E.Furneaux, V.M.Pudalov, and M. D'Iorio, Phys. Rev. B 50, 8039 (1994).

23 S.V.Kravchenko, W.E.Mason, G.E.Bowker, J.E.Furneaux, V.M.Pudalov, and M. D'Iorio, Phys. Rev. B 51, 7038 (1995).

24 M.Y.Simmons, A.R.Hamilton, M.Pepper, E.H.Linfield, P.D.Rose, D.A.Ritchie, A.K.Savchenko, and T.G. Griffiths, Phys. Rev. Lett. 80, 1292 (1998).

25 J.P.Eisenstein, L.N.Pfeiffer, and K.W.West, Phys. Rev. B 50, 1760 (1994).

26 J.P.Eisenstein, L.N.Pfeiffer, and K.W.West, Phys. Rev. Lett. 68, 674 (1992).

27 S.C.Dultz and H.W.Jiang, J. Phys. Soc. Jpn. 72, 674 (2003).

28 S.C.Dultz and H.W.Jiang, Phys. Rev. Lett. 84, 4689 (2000).

29 M. Seul and D. Andelman, Science 267, 476 (1995).

30 C. P. Lorenz, D. G. Ravenhall, and C. J.Pethick, Phys. Rev. Lett. 70, 379 (1993).

31 P. Fratzl, O. Penrose, and J. L. Lebowitz, J. of Stat. Phys. 95, 1429 (1999).

32 C. Kittel, Phys. Rev. 70, 965 (1946).

${ }^{33}$ L. Landau and E. Lifshitz, Electrodynamics of Continuous Media (Pergamon, New York, 1984).

34 J. Lorenzana, C. Castellani, and C. Di Castro, Phys. Rev. B 64, 235127 (2001).

35 J. Lorenzana, C. Castellani, and C. Di Castro, Phys. Rev. B 64, 235128 (2001).
36 J. Lorenzana, C. Castellani, and C. Di Castro, Europhys. Lett. 57, 704 (2002).

37 C. B. Muratov, Phys. Rev. E 66, 066108 (2002).

38 Throughout this work we call a metal a charged fluid which is compressible regardless of transport properties except when we obviously refer to transport as in "metal-insulator transition".

39 B. Spivak, Phys. Rev. B 67, 125205 (2003).

40 B. Spivak and S. A. Kivelson, Phys. Rev. B 70, 155114 (2004).

41 C. Ortix, J. Lorenzana, and C. Di Castro, in preparation (unpublished).

42 V. J. Emery, S. A. Kivelson, and H. Q. Lin, Phys. Rev. Lett. 64, 475 (1990).

43 S. A. Kivelson, V. J. Emery, and H. Q. Lin, Phys. Rev. B 42, 6523 (1990).

44 N. Cancrini, S. Caprara, C. Castellani, C. Di Castro, M. Grilli, and R. Raimondi, Europhys. Lett. 14, 597 (1991).

45 M. Grilli, R. Raimondi, C. Castellani, C. Di Castro, and G. Kotliar, Phys. Rev. Lett. 67, 256 (1991).

46 P. G. J. van Dongen, Phys. Rev. Lett. 74, 182 (1995).

47 S. Okamoto, S. Ishihara, and S. Maekawa, Phys. Rev. B 61, 451 (2000).

48 J. Lorenzana, C. Castellani, and C. Di Castro, Physica B 320, 56 (2002).

49 T. Ando, A.B.Fowler, and F.Stern, Rev. Mod. Phys. 54, 437 (1982).

50 M. Tinkham, Introduction to superconductivity (McGrawHill, New York, 1975).

51 W.R.Smythe, Static and dynamic electricity (McGrawHill, New York, 1975).

52 J. Lorenzana and G. Seibold, Phys. Rev. Lett. 89, 136401 (2002).

53 J.D.Jackson, Classical electrodynamics (J.Wiley and sons, New York, 1975).

54 E. Wigner, Phys. Rev. 46, 1002 (1934).

55 J. Shi, S. He, and X.C. Xie, Phys. Rev. B 60, R13950 (1999). 